\title{
O PROGRAMA SELETIVO MISTO (PISM) DA UFJF: UM ESTUDO DE CASO DO ATENDIMENTO ESPECIAL AOS CANDIDATOS COM DEFICIÊNCIA.
}

\author{
THE UFJF MIXED SELECTIVE PROGRAM: A CASE STUDY OF SPECIAL SERVICE \\ TO CANDIDATES WITH DISABILITIES.
}

\author{
Katiuscia Vargas Antunes ${ }^{13}$ \\ Mylene Cristina Santiago ${ }^{14}$ \\ Cassiano Caon Amorim ${ }^{15}$
}

\begin{abstract}
Resumo
A inclusão de pessoas com deficiência (PCD) no ensino superior vem ganhando espaço no campo da pesquisa em educação, particularmente, da educação especial e educação inclusiva. As políticas de democratização do acesso ao ensino superior e a política de reserva de vagas para pessoas com deficiência, que entrou em vigor em 2017, tem provocado um aumento gradativo de estudantes com deficiência nas universidades. Em função da relevância dessa temática, o presente artigo tem por objetivo analisar o atendimento especial oferecido pela Universidade Federal de Juiz de Fora (UFJF) no seu Programa de Ingresso Seletivo Misto (PISM). A partir do estudo de caso (YIN, 2001) dessa instituição, buscaremos traçar o percurso histórico do atendimento especial do PISM, ressaltando seu avanço, as fragilidades que ainda existem nesse atendimento e os desafios que se impõe às universidade no que se refere a promoção de acessibilidade às pessoas com deficiências nos seus processo seletivos. Os resultados desse estudo apontam para a necessária oferta dos serviços de atendimento especial durante a realização das provas do PISM, compreendendo que a acessibilidade nesse processo é o primeiro passo para que as pessoas com deficiência possam acessar o ensino superior. A reserva de vagas para esse público é um passo importante para a inclusão educacional, todavia, se os processos seletivos ainda continuarem oferecendo barreiras significativas para esses estudantes, não estaremos possibilitando o pleno direito à educação e aprendizagem aos estudantes com deficiência que intentam ingressar no ensino superior.
\end{abstract}

Palavras-chave: Pessoas com deficiência. Ensino superior. Inclusão em educação. Atendimento especial. Acessibilidade.

\section{Abstract}

The inclusion of people with disabilities at the University has been gaining ground in the field of research in education, particularly in special education and inclusive education.Policies to democratize access to the University and the policy of reserving places

\footnotetext{
13 Doutora e Mestre em Educação pela UERJ; graduada em Ciências Sociais pela UFJF. Professora Adjunta do Departamento de Educação da Universidade Federal de Juiz de Fora (UFJF). Email: katiuscia.vargas@educacao.ufjf.br.ORCID: 0000-0003-2861-551X

14 Doutora e Mestre em Educação pela UERJ; graduada em Ciências Sociais pela UFJF. Professora Adjunta do Departamento de Educação da Universidade Federal de Juiz de Fora (UFJF). Email:mylenesantiago87@gmail.com.ORCID:0000-0003-2769-8421

15 Doutor em Geografia Humana. Professor Associado do Departamento de Educação, da Universidade Federal de Juiz de Fora. Professora do Programa de Pós-graduação em Educação da UFJF). E-mail: cassianoamorim@hotmail.com ORCID:0000-0003-3326-8524
} 


\section{RevistAleph}

for people with disabilities, which came into force in 2017, have led to a gradual increase in students with disabilities in universities. Due to the relevance of this theme, this article aims to analyze the special care offered by the Universidade Federal de Juiz de Fora (UFJF) in its Mixed Selective Admission Program. Based on the case study (YIN, 2001) of this institution, we will seek to trace the historical trajectory of PISM's special service, highlighting its progress, the weaknesses that still exist in this service and the challenges that are imposed on the universities in terms of promotion accessibility for people with disabilities in their selection processes. The results of this study point to the necessary provision of special care services during the PISM tests, understanding that accessibility in this process is the first step for people with disabilities to access higher education. Reserving places for this audience is an important step towards educational inclusion, however, if the selection processes still continue to offer significant barriers for these students, we will not be enabling the full right to education and learning to students with disabilities who are trying to the university.

Keywords: People with disabilities. Higher education. Inclusion in education. Special care. Accessibility.

\section{Considerações iniciais}

A década de 1990 é considerada o marco na política educacional brasileira, que influenciada por movimentos internacionais tais como a Declaração de Jomtien (1990) e a Declaração de Salamanca (1994), resultou em decretos, resoluções, recomendações e diretrizes visando orientar os sistemas educacionais em uma perspectiva inclusiva. A Lei de Diretrizes e Bases da Educação Nacional em vigor (Lei 9.394/1996) explicita a opção por uma política pública inclusiva que prevê o acesso e a permanência dos alunos com deficiências, transtornos globais do desenvolvimento e altas habilidades/superdotação em todos os níveis e etapas da educação básica e do ensino superior, com o apoio de estratégias, recursos e metodologias diferenciadas e a previsão do atendimento educacional especializado.

Nas últimas três décadas os sistemas de ensino, principalmente públicos, vem se esforçando para garantir o direito à educação para todos. Esse processo não está consolidado e não podemos afirmar que existe instituição educacional inclusiva, visto que se trata de um processo infindável, que requer revisões sistemáticas e estudos contínuos, no sentido de conhecer cada aluno e aprender a superar as barreiras identificadas em escolas e instituições de ensino superior.

Todavia, os estudantes com deficiências, a despeito de todas as barreiras existentes na educação básica, estão ingressando no Ensino Superior, determinando que agora é o 


\section{RevistAleph}

momento de construir culturas, políticas e práticas de inclusão e garantir acesso e permanência com qualidade a todos aqueles que chegam aos cursos da Universidade Federal de Juiz de Fora.

Nesse artigo buscaremos apresentar e refletir uma experiência desenvolvida no Programa Seletivo Misto da UFJF (PISM/UFJF). Tal experiência configura-se numa possibilidade de ingresso à universidade, com atendimento especializado para candidatos que apresentam deficiências e outras circunstâncias e que necessitem de acessibilidade no sentido de diferenciar os meios para igualar os direitos.

O presente estudo foi balizado pelo estudo de caso (YIN, 2001), em que foram realizados os seguintes procedimentos: 1) levantamento e análise de documentos institucionais sobre o PISM, desde a sua criação; 2) levantamento e análise de bibliografia sobre a temática em estudo, apresentando o PISM como primeiro passo na garantia do direito ao acesso com acessibilidade na UFJF; 3) análise dos dados históricos sobre o atendimento especial do PISM, por meio dos relatórios elaborados pela coordenação deste serviço.

Na primeira seção deste artigo apresentaremos uma breve contextualização da UFJF e do PISM, situando o leitor sobre as características da universidade e sua relevância para Juiz de Fora e seu contexto regional. Sobre o PISM, apresentaremos dados gerais acerca da abrangência desse processo seletivo e seus impactos na sociedade. Na segunda seção, apresentaremos o levantamento e análise de bibliografia produzida

Em seguida nos deteremos na descrição e análise do atendimento especial do PISM e seus desdobramentos no que se refere à promoção da acessibilidade para os candidatos com deficiência e outras necessidades especiais, considerando que esse é um primeiro movimento no sentido de garantir o acesso das pessoas com deficiência ao ensino superior.

Por fim, apresentaremos algumas considerações que este estudo de caso nos permitiu fazer, numa tentativa de apontar caminhos possíveis e desafios que estão colocados para o ensino superior, quando tratamos da inclusão de pessoas com deficiência.

\section{A Universidade Federal de Juiz de Fora e o seu Programa Seletivo de Ingresso Misto (PISM)}

Criado no ano de 1999, através da Resolução n. 18/99 do Conselho Setorial de Graduação (CONGRAD), o PISM se caracteriza por ser um processo de ingresso seriado, 


\section{RevistAleph}

gradual e cumulativo no qual o candidato, ao final de cada ano do ensino médio, realiza uma prova, sendo Módulo I, Módulo II e Módulo III. O somatório das notas dessas avaliações compõe a nota final e classificatória o que possibilita ao candidato concorrer a uma vaga para os cursos de graduação da UFJF. Até o ano de 1999 a única forma de ingresso na universidade era o vestibular tradicional. Atualmente, a política de ingresso na UFJF apresenta duas possibilidades aos candidatos: o PISM e Sistema de Seleção Unificada (SISU/ENEM). As vagas dos cursos de Graduação são divididas $50 \%$ para cada um desses processos.

Enquanto programa de ingresso, ainda é importante reiterar que o PISM funciona articulado com o SISU, que é um sistema cuja gestão é feita pelo Ministério da Educação. O SISU permite um amplo acesso a diferentes camadas da população porque utiliza a nota do Enem. O Enem possibilita a todos que se submeteram ao exame, usar as notas obtidas naquele ano específico para o ingresso nas universidades.

Com o objetivo de possibilitar um acesso mais democrático ao processo seletivo, para a realização do PISM, a UFJF conta com um significativo número de escolas do município de Juiz de Fora. Mapeando as demandas de inscrições de outras localidades, a universidade instituiu locais de realização dos exames também nas cidades de Volta Redonda (RJ), Muriaé (MG) e Governador Valadares (MG). Em 2019 a aplicação do PISM foi expandida para Petrópolis porque foi verificado que há um considerável aumento do número de inscrições de estudantes do Estado do Rio de Janeiro, principalmente da região serrana e região metropolitana do Rio.

Uma das características de um processo de seleção de candidatos feito de maneira seriada, ano a ano, está relacionada à preparação dos estudantes. As provas de seleção são elaboradas com os conteúdos presentes nos editais, específico de cada ano. Dessa maneira, permite aos estudantes, professores e familiares, acompanhar a evolução dos estudantes nas provas.

Nos últimos anos houve um crescimento muito significativo da procura pelo PISM. Dados apresentados pela Comissão Permanente de Processos Seletivos (COPESE) demonstram um maior interesse dos estudantes pelo PISM. 


\section{RevistAleph}

Quadro 1 - NÚMERO DE CANDIDATOS INSCRITOS NO PISM ENTRE OS ANOS DE 2016 E 2020

\begin{tabular}{|c|c|}
\hline ANO & TOTAL DE CANDIDATOS INSCRITOS \\
\hline 2016 & 24.184 \\
\hline 2017 & 26.059 \\
\hline 2018 & 29.626 \\
\hline 2019 & 34.754 \\
\hline 2020 & 40.160 \\
\hline
\end{tabular}

Fonte: COPESE

Enquanto uma política de ingresso, acreditamos que ela atenda aos princípios do acesso democrático ao ensino superior, porque há possibilidade de isenção de taxa, conforme legislação e a universidade tem ampliado a possibilidade desses estudantes realizarem as provas em outros lugares do território de Minas Gerais e do Estado do Rio de janeiro. Somamos a isso, o atendimento especial ofertado no PISM aos candidatos que fizerem a sua requisição.

Desde a sua criação o PISM passou por muitas modificações com o objetivo de aprimorar o processo. Dentre as mudanças ocorridas no processo, nos cabe ressaltar a oferta do atendimento especial.

O atendimento especializado no PISM: primeiro passo para possibilitar o acesso de Pessoas Com Deficiência (PCD) à universidade

O atendimento especial do PISM é um serviço que objetiva promover condições de acessibilidade para que os candidatos com deficiência ou outras necessidades explicitadas no edital possam realizar as provas em condições adequadas e adaptadas às suas necessidades especiais. Os candidatos que podem solicitar o atendimento especial são aqueles considerados público-alvo da educação especial, conforme descrito na Política Nacional de Educação Especial na Perspectiva da Educação Inclusiva (BRASIL, 2008). Além desses, candidatos que apresentam problemas de saúde que demandam maiores cuidados, lactantes, candidatos sabatistas (que por questões religiosas só podem realizar a prova após o por do sol de sábado) podem acessar o atendimento especial. No escopo deste artigo daremos destaque ao atendimento especial destinado às pessoas com deficiência. 


\section{RevistAleph}

A oferta de atendimento especial no PISM intenta cumprir os dispositivos legais que regulamentam as políticas de acesso ao ensino superior, a saber, o Aviso Circular no 277/MEC/GM, de 08 de maio de 1996 (MEC, 1996), e Lei Brasileira de Inclusão da Pessoa com Deficiência (Estatuto da Pessoa com Deficiência) - Lei no 13.146, de 06 de julho de 2015 (BRASIL, 2015). Tais documentos, entre outras determinações, indicam que as instituições de ensino superior devem oferecer condições básicas para que os candidatos com deficiência participem dos processos seletivos com condições de acessibilidade. Dentre as determinações, estão: instalar Banca Especial para conduzir o atendimento especial; disponibilizar o edital em Libras; informar, no edital, os recursos de acessibilidade e de tecnologia assistiva que poderão ser utilizados durante a realização das provas, bem como disponibilizar um formulário para que o candidato registre as suas necessidades; oferecer provas acessíveis de acordo com a deficiência de cada candidato (deficiência visual e/ou auditiva, por exemplo); permitir dilatação do tempo para a realização das provas, de acordo com a demanda dos candidatos; flexibilizar os critérios de correção das provas discursivas e da redação dos candidatos com deficiência, principalmente auditiva, entre outras (BRASIL, 2015; MEC, 1996).

Em sua pesquisa de mestrado VEIGA (2020) realizou um estudo sobre o atendimento especial no PISM, demonstrando através de um cuidadoso levantamento bibliográfico e uma pesquisa documental que não existia na UFJF nenhum estudo voltado para analisar essa temática. Em seus achados, Veiga (2020) constatou que as solicitações pelo atendimento especial estão previstas nos editais do PISM desde a sua primeira edição, no ano 2000, quando contemplava apenas os candidatos com "deficiência física" e aqueles em condições graves de saúde. Além disso, as solicitações deveriam ser requeridas, presencialmente, junto à Coordenação dos Programas de Ingresso (COPESE/UFJF, 1999). No ano seguinte o procedimento seguiu os mesmos moldes dos anos anteriores.

No ano de 2002 houve a primeira modificação no atendimento especial, expandido esse serviço a todos os candidatos com deficiência e não apenas aos com deficiência física. Entre os anos 2002 e 2006, o atendimento especial permaneceu com as mesmas características no que se refere aos seus destinatários. A principal diferença foi que ao invés dos candidatos solicitarem esse atendimento à COPESE, passou a fazê-lo junto a Central de Atendimentos da UFJF. 


\section{RevistAleph}

Importa ressaltar que até o ano de 2006 não havia sido constituída formalmente uma equipe de coordenação do atendimento especial e os serviços oferecidos aos candidatos para a realização das provas se restringiam a oferta de salas e mobiliário acessíveis, oferta de ledor e transcritor para candidatos com deficiência visual e horário de prova especial para os candidatos sabatistas. Nesse contexto ainda era um serviço bastante precário.

Foi no ano de 2007 que o atendimento especial do PISM passou a conferir uma especificidade de gestão no processo seletivo, sendo constituída, à época, uma equipe para coordenar esse trabalho. A partir desse ano, a forma de solicitação desse serviço, a diversidade de ofertas de recursos de acessibilidade e os registros em relação aos candidatos em atendimento especial passaram a ser melhor organizados e sistematizados.

O levantamento do histórico do atendimento especial do PISM realizado por Veiga (2020) demonstra uma evolução significativa, do ponto de vista quantitativo, no número de candidatos que solicitam esse serviço.

Com os relatórios elaborados pela coordenação do atendimento especial a partir de 2007 foi possível realizar um histórico da sua evolução. Veiga (2020) identificou que na edição do PISM de 2007 os seguintes dados: 30 candidatos solicitaram atendimento especial, sendo que destes 11 eram sabatistas e apenas 06 eram pessoas com deficiência. Os demais eram candidatos que apresentavam alguma condição de saúde que demandava maiores cuidados. Outro aspecto relevante é o número de pessoas envolvidas no atendimento especial. Entre equipe de coordenação, fiscais e aplicadores de prova foram envolvidas 38 pessoas. A única orientação que esses trabalhadores receberam ocorreu em duas reuniões, onde foram passadas informações básicas sobre a condição de cada candidato.

No ano 2008, sob a coordenação da mesma equipe, o atendimento especial atendeu 39 candidatos. Desse total, foram identificadas que 28 eram pessoas com deficiência. Tal fato pode ter se dado em virtude das modificações ocorridas no atendimento especial, oferecendo aos candidatos mais recursos de acessibilidade, com a disponibilização de um formulário para solicitação do atendimento especial no ato da inscrição. Além disso, o edital trouxe maiores esclarecimentos quanto à documentação necessária à solicitação do atendimento especial (UFJF, 2008). 


\section{RevistAleph}

Em 2009, pelos registros levantados na COPESE, o número de solicitações de atendimento especial relativas às pessoas com deficiência foi 19.

No período entre 2010 e 2017, sob uma nova coordenação, o atendimento especial no PISM manteve-se nos mesmos moldes dos anos anteriores, entretanto, segundo levantamento realizado por Veiga (2020), não foram encontrados relatórios com registros dos atendimentos. Esse gap, infelizmente, não nos permite ter uma dimensão mais adequada do quanto foi crescendo a demanda por esse serviço.

A retomada dos registros ocorreu em 2014, contabilizando um total de 49 solicitações por atendimento especial, representa um número significativamente maior do que as 19 solicitações em 2009. O número de solicitações por atendimento especial nos anos subsequentes até a última edição em 2020 teve um crescimento expressivo.

Entre os anos 2014 e 2020 temos presenciado um aumento crescente de demanda pelo atendimento especial do PISM, conforme tabela abaixo.

Tabela 2 - DEMANDAS PELO ATENDIMENTO ESPECIAL DO PISM 2014 -2020

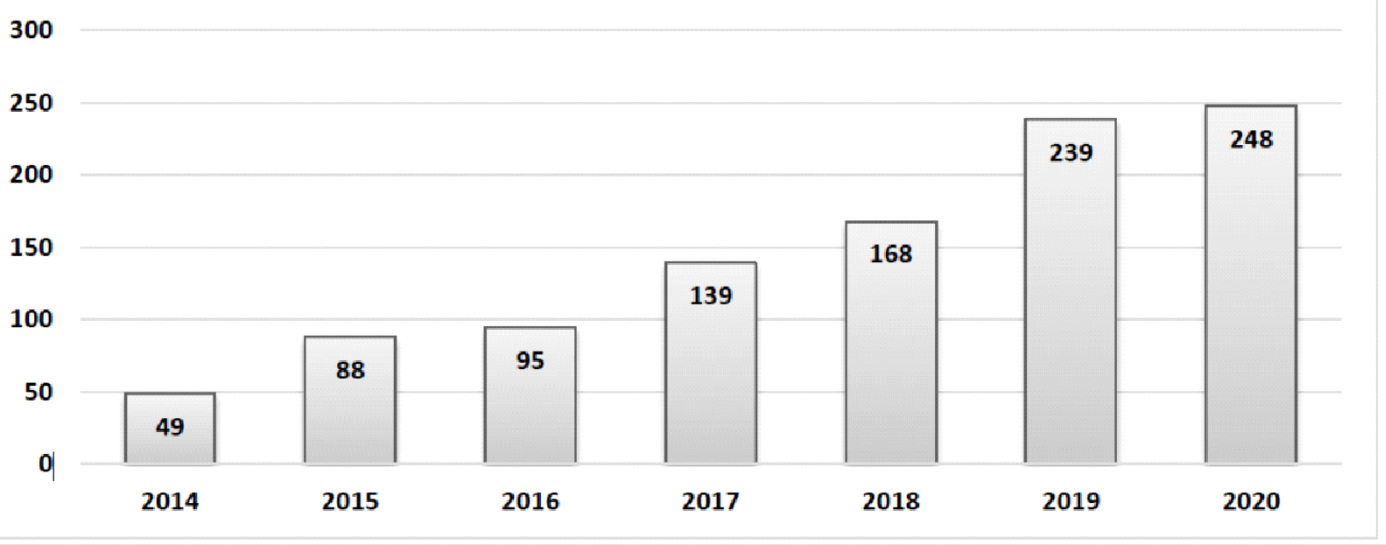

Fonte: VEIGA, 2019.

Do ponto de vista qualitativo, as maiores mudanças da oferta do atendimento especial ocorreram a partir de 2016. Conforme informações retiradas dos relatórios elaborados pela coordenação do atendimento especial, o formulário de solicitação de atendimento especial foi aperfeiçoado, com campos específicos para os candidatos assinalarem suas necessidades especiais e recursos necessários para a realização das provas (UFJF, 2016). Quanto aos recursos disponíveis para a realização das provas, foram disponibilizados: dilatação de tempo para a realização das provas, ledor, transcritor, prova em braile, prova ampliada, lupa, reglete e punção, máquina de perkins, soroban, cubaritmo, intérprete/tradutor de Libras, possibilidade do uso de aparelho auditivo ou de implante (c) (i) (2) 


\section{RevistAleph}

coclear, salas acessíveis com rampas ou elevadores, carteiras e cadeiras em tamanhos maiores, mesa adaptada para uso de cadeira de rodas, suporte para provas e computador. Além desses recursos, os candidatos puderam registrar outras demandas e demais observações sobre a sua condição especial e/ou deficiência (UFJF, 2016).

Quanto à equipe que atuou no atendimento especial no ano de 2016, diferente do que aconteceu nos anos anteriores, foram incorporados profissionais especializados para atender os candidatos. Compôs a equipe, além da coordenação e fiscais/aplicadores, 03 fonoaudiólogos, 02 pedagogos especialistas em educação especial, 01 psicólogo. As reuniões de orientação que aconteceram com a equipe nos anos anteriores, deixaram de existir, ficando a cargo da coordenação de cada setor de realização das provas, o fornecimento de orientações para os trabalhadores.

O aumento da demanda por atendimento especial no PISM foi impulsionando mudanças no sentido de aperfeiçoar o processo de trabalho da equipe, desde modificações significativas no edital, que passou a ter, a partir de 2017, um capítulo dedicado ao atendimento especial, até à informatização do processo de solicitação desse atendimento, que desde 2018 vem sendo realizado on line, por um sistema elaborado especialmente para este fim. Outra mudança significativa no processo se deu em relação à coordenação do atendimento especial, que deixou de ser realizada por servidores ocasionalmente convidados para exercer essa função e passou a ser uma das atribuições do Núcleo de Apoio à Inclusão (NAI), criado em 2018 e, desde então, responsável pela coordenação do processo. Os recursos e apoios oferecidos aos candidatos com deficiência que vinham sendo disponibilizados desde 2016 se mantiveram e na última edição do PISM 2020, foi acrescentada a possibilidade dos candidatos cegos realizarem a prova no computador, com a utilização de softwares de leitura. Além disso, todos os candidatos que solicitam atendimento especial tem a dilatação do tempo para a realização da prova de uma hora a mais.

No ano de 2018 a formação da equipe que atua no atendimento especial foi retomada. A coordenação organiza um encontro de formação, onde esclarece como se processa o atendimento especial e quais são as especificidades desse trabalho. Nessa mesma formação também são passadas orientações aos trabalhadores que atuam como ledores e transcritores. Importa destacar que a retomada do processo de formação da equipe foi avaliada de forma positiva, entretanto, ainda é insuficiente, posto que se 


\section{RevistAleph}

constitua de apenas um dia. Aliás, essa foi uma das fragilidades que Veiga (2020) apontou. Ao entrevistar fiscais que atuaram na última edição do PISM, a autora constatou que os entrevistados, de forma unânime, consideraram que um dia de formação não é suficiente e que seria necessário um curso de curta duração para capacitar melhor a equipe, em especial àqueles que atuam como ledores e transcritores.

Frente ao histórico do atendimento especial do PISM é evidente que o crescimento significativo da demanda por esse serviço, juntamente com a postura assumida pelo NAI na condução desse processo vem provocando a instituição no sentido de, a cada ano buscar novas formas de proporcionar maior acessibilidade aos candidatos com deficiência. Os limites para que o programa de seleção misto seja plenamente acessível são grandes e se evidenciam, sobretudo, no formato da prova.

Sobre o formato da prova e seu processo de elaboração cabe ressaltar que, a despeito de todos os recursos oferecidos aos candidatos com deficiência, as provas ainda representam uma barreira para os candidatos, quando trazem em seu corpo textos muitos longos, o que para candidatos com deficiência intelectual ou transtorno do espectro autista, por exemplo, representa um dificultador; muitos gráficos e figuras, o que para pessoas cegas representa uma barreira, mesmo com os recursos oferecidos; questões dissertativas cujas respostas em língua portuguesa, representam uma dificuldade para os estudantes surdos, considerando que para esses sujeitos o português é a segunda língua e a fluência na escrita é bem diferente da de um ouvinte... Esses são apenas três exemplos que ilustram alguns desafios enfrentados pelos candidatos. Além disso, durante o processo de elaboração das provas, não há envolvimento da equipe de coordenação do atendimento especial com as bancas de elaboração de questões. Essa é uma grande fragilidade, considerando que se houvesse uma articulação entre as equipes no momento de elaboração da prova, os professores poderiam ser orientados a elaborar questões dentro da lógica do desenho universal para a aprendizagem (NUNES; MADUREIRA, 2015), de modo a ampliar acessibilidade curricular, minimizando possíveis barreiras como as que citamos anteriormente.

Sabemos do grande desafio que é elaborar uma prova, em um processo seletivo como o PISM, em que milhares de jovens então envolvidos. Sabemos ainda que quando entra em jogo a acessibilidade para as pessoas com deficiência os desafios se potencializam se considerarmos as especificidades que os candidatos com deficiência apresentam. Esse é 


\section{RevistAleph}

um dilema para o qual não temos, ainda, uma resposta. O que sabemos é que essa é uma discussão que precisa ser realizada pelas instituições de ensino superior, que também tem os seus processos seletivos específicos.

\section{A Universidade Federal de Juiz de fora e os processos de inclusão no contexto da permanência}

Após o acesso do estudante com deficiência na UFJF, deparamo-nos com o desafio de garantir sua permanência. Os desafios são múltiplos e interseccionais; podem estar associados a barreiras físicas e arquitetônicas, visto que o campus da UFJF está situado em um espaço não plano e que suas edificações necessitam de adaptações e manutenções constantes. A UFJF dispõe de transporte coletivo que circulam no campus e possibilitam maior mobilidade da comunidade acadêmica, no entanto, nem todos os veículos são adaptados. As barreiras curriculares e comunicacionais têm sido minimizadas com contratação de intérpretes de Libras para alunos surdos e acompanhamento acadêmico para estudantes que necessitam de forma contínua de adaptações de recursos. A diretoria responsável pela imagem institucional tem se empenhado em produzir vídeos com tradução em Libras para garantir o direito à informação aos estudantes e servidores surdos. A Pró-Reitoria de Graduação e Diretoria de Ações Afirmativas e o Núcleo de Apoio à Inclusão vêm se empenhando em garantir condições de inclusão tecnológica, adquirindo equipamentos que permitam maior interação e superação das barreiras de participação e de aprendizagem.

E quanto às barreiras atitudinais? Essas são as mais subjetivas e complexas de superar, visto que estão vinculadas aos valores e concepções de cada pessoa em relação ao outro. Tem sido comum ouvirmos questionamentos sobre a finalidade de determinada disciplina para estudantes com deficiência ou ainda comentários sobre o despreparo do professor do ensino superior para lidar com essa problemática. Por outro lado, deparamonos com colegas dotados de grande sensibilidade pedagógica e que desejam aprimorar seus conhecimentos e repertórios didáticos para oferecer condições concretas de aprendizagem para todos os estudantes.

Como se vê, as realidades são múltiplas, nesse sentido temos buscado disponibilizar condições de oferecer suporte aos docentes e técnicos. Sabemos que cursos de formação 


\section{RevistAleph}

continuada são importantes no processo de inclusão, mas os professores têm apresentado demandas urgentes na busca de obter respostas e soluções imediatas para o estudante que se encontra na sala de aula, para isso temos nos colocado à disposição para conversas com potencial formativo e possíveis estudos de caso, envolvendo professores e coordenação de curso.

Cientes de que tal estratégia pode não ser suficiente, no contexto de uma universidade com quase 20 mil estudantes, reconhecemos a necessidade de empenho na construção de políticas, culturas e práticas de inclusão no ensino superior e na articulação de propostas intersetoriais que identificam barreiras institucionais, para então saná-las coletivamente. Esse tem sido o principal objetivo do Núcleo de Apoio a Inclusão, criado no segundo semestre de 2018.

O que seria a construção de culturas, políticas e práticas? Tais dimensões são apresentadas no Index para Inclusão (BOOTH; AINSCOW, 2011), material que tem servido de base para a construção de projetos de pesquisa, ensino e extensão. Vale dizer que, em termos práticos, o Index pode ser adaptado a qualquer instituição e utilizado com qualquer ator social. No contexto da UFJF, podemos considerar que a dimensão das culturas referese a valores que temos e justificativas que usamos no combate às exclusões. No que tange ao âmbito das políticas faz referência não apenas às conhecidas políticas públicas, como também abrange toda intenção expressa que tem por objetivo orientar e organizar ações. Políticas refletem decisões tomadas e geralmente implicam em planos de ação. A das práticas diz respeito às práticas e ações pessoais e sociais propriamente ditas, se referem a tudo que se faz no cotidiano das instituições (SANTIAGO; SANTOS, 2015).

Nossa proposta com base nesse referencial têm sido formar equipes de trabalhos intersetoriais e promover discussões no intuito de demonstrar que o processo de inclusão demanda envolvimento e responsabilidade coletiva. Muitas ações têm sido realizadas no cotidiano de cada sala de aula, de cada departamento e instituição, a tarefa que se coloca é como fazer com que ações isoladas se tornem ações institucionais voltadas para a inclusão? Ainda precisamos caminhar bastante!

\section{Considerações finais}




\section{RevistAleph}

O trabalho que vem sendo realizado pela UFJF no atendimento especial do PISM tem permitido que a instituição intensifique as reflexões sobre a inclusão no ensino superior. As políticas de ingresso são, apenas, parte do desafio de constituição da cultura de inclusão em cursos de Graduação e Pós-Graduação. Como já destacado, não foi possível, por diferentes limitações, atender a todas as demandas apresentadas por candidatos e candidatas que apresentam diferentes situações ao atendimento especial do PISM. Tais limitações vinculam-se, igualmente, a diversas questões da gestão acadêmicas e financeiras das IES.

Os avanços destacados nesse estudo de caso corroboram com outras ações inclusivas, quais sejam: reserva de vagas específicas para surdos no curso de letras libras; aquisição de equipamentos para ampliação do atendimento a estudantes com deficiência; criação do núcleo de apoio à inclusão; criação do setor de intérpretes em Libras; organização de formação continuada a docentes e técnicos, entre outros.

No atendimento especial às pessoas com deficiência há limitações que ainda precisam ser superadas. A aquisição de equipamentos e o desenvolvimento de novas metodologias para desenvolvimento das avaliações são necessários. É importante também ampliar a capacitação das pessoas envolvidas no processo, principalmente as que atuam fora do Campus sede da UFJF.

Nesse sentido, a oferta do atendimento especial, tem se configurado também num importante momento formativo. Apesar da necessidade de ampliação da formação de quadros para atendimento das demandas no ato da realização das provas, as capacitações já realizadas têm mobilizado servidores e estudantes, com sensibilidade e interesse para a cultura da inclusão.

Também, como já mencionado, a oportunidade no contato com pais e responsáveis no dia de realização das provas, tem possibilitado a UFJF um maior conhecimento das realidades dos e das estudantes que requerem atendimento especial e que ingressarão na universidade. Isso tem possibilitado ações de planejamento pedagógico, financeiro e administrativo em relação às políticas de ingresso e permanência de estudantes com deficiência na UFJF.

Para concluir, o processo de ingresso praticado na UFJF tem possibilitado pensar que as estratégias de acesso precisam ampliar as políticas de permanência com condições de aprendizagem e participação para todos os estudantes, assim como estabelecer culturas e 


\section{RevistAleph}

práticas orientadas em uma perspectiva intersetorial que nos possibilitam trabalhar de forma colaborativa, com a compreensão de que o processo de inclusão no ensino superior é responsabilidade de toda a comunidade acadêmica. 


\section{Referências}

BOOTH, T.; AINSCOW, M. Index para a inclusão em educação: desenvolvendo a participação e a aprendizagem nas escolas. Rio de Janeiro: LaPEADE, 2011.

BRASIL, Lei no 9.394, de 20 de dezembro de 1996. Estabelece as diretrizes e bases da educação nacional. Diário Oficial da União, Brasília, DF, 21 dez. 1996.

BRASIL. Lei no 13.146, de 6 de julho de 2015. Institui a Lei Brasileira de Inclusão da Pessoa com Deficiência (Estatuto da Pessoa com Deficiência). Diário Oficial da União, Brasília, DF, 06 jul. 2015.

BRASIL. Ministério da Educação (MEC). Aviso Circular no 277, de 08 de maio de 1996. Brasília, DF, 08 maio 1996. Disponível em: http://portal.mec.gov.br/seesp/arquivos/pdf/aviso277.pdf. Acesso: em 12 ago. 2019.

BRASIL. Política Nacional de Educação Especial na Perspectiva da Educação Inclusiva. Brasília, DF, 07 jan. 2008. Disponível em: http://portal.mec.gov.br/arquivos/pdf/politicaeducespecial.pdf. Acesso em: 02 ago. 2019.

NUNES, C., MADUREIRA, I. Desenho Universal para a Aprendizagem: Construindo práticas pedagógicas inclusivas, p. $126-143,2015$. Disponível em:

http://www.scielo.mec.pt/pdf/inp/v5n2/v5n2a08.pdf Acesso em: 25 de mar. 2020.

SANTOS, M. C.; SANTOS, M. P. dos. Planejamento de Estratégias para o Processo de Inclusão: desafios em questão. Revista Educação \& Realidade, Porto Alegre, v. 40, n. 2, p. 485-502, abr./jun. 2015. 485 http://dx.doi.org/10.1590/2175-623645248

UNIVERSIDADE Federal de Juiz de Fora. Edital Módulo I do Pism. Juiz de Fora, MG, 27 set. 1999.

UNIVERSIDADE Federal de Juiz de Fora. Edital Concurso Vestibular 2009 Programa de Ingresso Seletivo Misto - Pism. Juiz de Fora, MG, 01 set. 2008b. Disponível em:

http://www.ufjf.br/antenado/files/2011/05/Edital_Vestibular-_2009.pdf. Acesso em: 16 set. 2019.

UNIVERSIDADE Federal de Juiz de Fora. Edital no 04/2016 Programa de Ingresso Seletivo Misto Pism. Juiz de Fora, MG, 06 set. 2016b. Disponível em: https://www2.ufjf.br/copese/wpcontent/ ploads/sites/42/2016/08/Edital-PISM-2017-Final2.pdf. Acesso em: 18 set. 2019.

UNESCO. Declaração de Salamanca e linha de ação sobre necessidades educativas especiais. 1994. Disponível em: http://www.educacaoonline.pro.br, acessado em: 20 nov. 2019.

VEIGA, C. L. da. Compreendendo os desafios do Atendimento Especial do Programa de Ingresso Seletivo Misto (Pism) da Universidade Federal de Juiz de Fora (UFJF). 2020. 155f. Dissertação (Mestrado em gestão e Avaliação da Educação Pública) - Centro de Políticas Públicas e Avaliação da Educação. Universidade Federal de Juiz de Fora, Juiz de fora, 2020.

WCEFA - CONFERÊNCIA MUNDIAL DE EDUCAÇÃO PARA TODOS. Declaração mundial sobre a educação para todos e Plano de ação para satisfazer as necessidades básicas de aprendizagem. Jomtien, Tailândia: março de 1990.

YIN, R. Estudo de caso: planejamento e métodos. 2 ed. Porto Alegre: bookman, 2001.

Data do envio: $03 / 04 / 2020$

Data do aceite: 13/05/2020. 